\title{
Cyber Physical Information Systems
}

\author{
Christian Becker \\ University of Mannheim \\ christian.becker@uni- \\ mannheim.de
}

\author{
Jörg Nolte \\ Brandenburgische Technische \\ Universität Cottbus-Senftenberg \\ joerg.nolte@b-tu.de
}

\author{
Matti Rossi \\ Aalto University School of \\ Business \\ $\underline{\text { matti.rossi@aalto.fi }}$
}

\author{
Wolfgang Schröder-Preikschat \\ Friedrich-Alexander-Universität Erlangen-Nürnberg (FAU) \\ wosch@,cs.fau.de
}

The proliferation of sensors, actuators and embedded processor platforms leads to information systems which are able to directly link to the physical world. Based on sensor data accurate and timely reactions become possible and allow to change processes as well as the physical world via actuators.

The problems of designing, deploying and maintaining cyber physical systems are complex and are well reflected by the papers in this track.

"Dynamic Composition of Cyber-Physical Systems" presents an approach that allows programmers to add a specification of the designed cyber physical system while they are creating it. Thus, at further phases, e.g., deployment when the system is connected with other systems in the physical environment and during runtime, this information can be used to check conformance to the initial specification.

Engineering cyber physical systems does not only require proper specifications but also suitable processes and tools to facilitate the construction process. "How to connect design thinking and cyberphysical systems: the $\mathrm{s}^{*} \mathrm{IoT}$ conceptual modelling approach" shows an approach how a well established design process from the enterprise world can be tailored to support the integration of cyber physical systems.

At runtime, cyber physical systems generate vast amounts of data, that can be used for monitoring purposes, optimization and fine granular accounting. Thus, capturing and communicating state is a must in such systems. "A Case for Integrated Data Processing in Large-Scale Cyber-Physical Systems" presents a general data processing architecture and most remarkable a use case analysis from a concrete realization.
During the communication between entities in cyber physical information systems, the data has to be processed and stored. The approach in the last paper of our minitrack is focusing on the data tier and strives for predictable storage in embedded systems. Thus, "Towards Low-Jitter and Energy-Efficient Data Processing in Cyber-Physical Information Systems" is contributing to the technical realization of efficient data processing in cyber physical information systems.

We arranged the presentation of the papers from a modeling/architectural perspective to technical aspects of communication and storage. 